\title{
Study of Optimal Real-time Polymerase Chain Reaction Chip by Comparison of Coating Reflective Materials
}

\author{
Ji-Soo Hwang, ${ }^{1,2}$ Bo-Jun Kim, ${ }^{1,2}$ Jong-Dae Kim, ${ }^{1,2}$ Yu-Seop Kim, ${ }^{1,2}$ \\ Hye-Jeong Song, ${ }^{1,2}$ and Chan-Young Park ${ }^{1,2^{*}}$ \\ ${ }^{1}$ Department of Convergence Software, Hallym University, \\ 1, Hallimdaehak-gil, Chuncheon-si, Gangwon-do 24252, Republic of Korea \\ ${ }^{2}$ Bio-IT Research Center, Hallym University, \\ 1, Hallimdaehak-gil, Chuncheon-si, Gangwon-do 24252, Republic of Korea \\ (Received May 31, 2018; accepted October 18, 2018)
}

Keywords: real-time polymerase chain reaction, fluorescence detection, relative gap, microfluidic channel, PCR chip, lab-on-a-chip

The real-time polymerase chain reaction (RT-PCR) test chip employed in previous experiments used a matte black printed circuit board (PCB) substrate printed with a white silk legend on a heating pattern. However, when analyzing the fluorescence detection results, the chip needed to be replaced with a coating material with a high reflection efficiency because of a large variation in brightness. Therefore, in this work, chips using a PCB substrate coated with various kinds of materials are manufactured, and the relative gains of these chips are compared to select a chip showing the most optimal reflection efficiency. As a result, the reflection efficiency of the PCR chip using a silver-coated PCB substrate was found to be the best.

\section{Introduction}

The polymerase chain reaction (PCR) is a molecular biology technique that amplifies small amounts of genetic samples in large quantities. This technique is used to amplify nucleic acids such as DNAs or RNAs to diagnose various genetic diseases.

In addition, it can be applied to bacteria, viruses, and fungi to diagnose infectious diseases. The technology derived here plays an important role in many fields, such as medical care, criminal investigation, and archeology. ${ }^{(1)}$

Since the existing PCR technique is unable to determine the success of the amplification by only amplifying the DNAs or the RNAs, it was necessary to perform an electrophoresis test to verify the amplification of the DNA. Since additional experiments after the PCR test are required, not only is the entire experiment time prolonged, but also contamination may occur during the electrophoresis.

*Corresponding author: e-mail: cypark@hallym.ac.kr https://doi.org/10.18494/SAM.2019.2101 
Real-time PCR (RT-PCR), which compensates for these problems, enables the quantitative analysis of nucleic acid amplification by monitoring the intensity of fluorescence detected in real time, in contrast to conventional PCR. ${ }^{(2-4)}$

In previous studies, we made PCR chips from printed circuit boards (PCBs), $50-\mu \mathrm{m}$-thick packing tape, $400-\mu \mathrm{m}$-thick double-sided tape, and 500- $\mu \mathrm{m}$-thick plastic covers and performed RT-PCR tests using a camera-based RT-PCR system.

The analysis of changes in the fluorescence brightness of images obtained as a result of RTPCR showed a tendency similar to those of commercial RT-PCR. Experimental results showed that it is possible to quantitatively analyze the change in fluorescence brightness. ${ }^{(5-7)}$

The PCR chip employed in previous experiments used a matte black PCB to minimize light reflections and a white silk legend printed on the heater pattern part of the substrate. In the experiments using silk chips, the changes in fluorescence brightness were similar to those of the commercial RT-PCR, but the fluorescence intensity averages of the images showed large deviations. In fact, when we visually compared several PCB substrates printed with silk legends produced by the PCB manufacturer, we found that the white silk color and density deviations were very large.

The accuracy and reliability of fluorescence detection in RT-PCR are very important. Therefore, fluorescence detection performance can be improved if an optimal chip can be manufactured by selecting and coating an optimal material having excellent reflection efficiency.

In this study, we fabricated chips using PCB substrates coated with four different materials. Then, distilled water with a slight amount of fluorescent material and fluorescein amidite (FAM) reagent showing fluorescence brightness similar to the amplified DNA were injected into each chip. A blue LED was diagonally illuminated and captured with a digital single-lens reflex (DSLR) camera to compare and analyze the average fluorescence brightness of the reaction chamber area.

At the beginning of the experiment, a photograph was taken at the same shutter speed and then the image was analyzed. Each time a reagent was injected, the chip was detached from the connector. For this reason, the position of the chip changed slightly, making it difficult to analyze the same area for the same chip. As a result of the initial experimental analysis, the relative gain of the tin-coated chip was the largest, followed by that of the gold-coated chip. However, silver-coated and silk-coated chips could not be used to calculate the maximum relative gain value. Therefore, additional experiments were needed. In later experiments, the chips were fixed while the reagents were changed so that the positions of the chips did not change when injected. Also, to obtain the maximum relative gain, silver- and silk-coated chips were photographed with a faster shutter speed. As a result of image analysis, the maximum relative gain was obtained for each type of chip. In the experiment, the silver-coated chip showed the maximum relative gain. If a PCR chip using a silver-coated substrate is used, accurate quantitative analysis is expected to be possible, unlike when using the previous PCR chips. 


\section{Materials and Methods}

\subsection{Fluorescence detection system}

Figure 1 shows the fluorescence detection system used in the experiment. This system is capable of RT-PCR.

In order to amplify the DNA, the sample is heated and cooled repeatedly. The local system that realizes this process, shown in Fig. 1, uses a PIC18F4550 microprocessor to control the analog-to-digital converter (ADC) and pulse width modulation (PWM) during RT-PCR. This temperature control mechanism includes a process of heating the PCR chip and a cooling process using a fan. The PCR protocol, which is set up for the amplification of DNAs through the USB interface, is configured to be controlled by a PC.

In addition to the local system, the system includes an illumination part and a fluorescence detection part. The illumination consists of a 5-mm-diameter blue LED (Broadcom Limited) with 9600 mcd brightness and an excitation filter (Edmund Optics Co. Ltd.).

The fluorescence detection part was composed of a DLSR camera, an extension tube for magnification, and an emission filter (Edmund Optics Co. Ltd.) for the fluorescence detection of a specific wavelength band.

When RT-PCR is performed using this system, the entire system is used. However, in this experiment, we injected distilled water and a FAM reagent into four types of chips in order to select the optimum chip material by comparing the signal-to-noise ratios of the chips without performing RT-PCR. Then, the blue LED was illuminated diagonally, and the reaction chamber portion of the PCR chip was photographed with a DSLR camera to compare the fluorescence brightness differences of the captured chip images. ${ }^{(8-13)}$

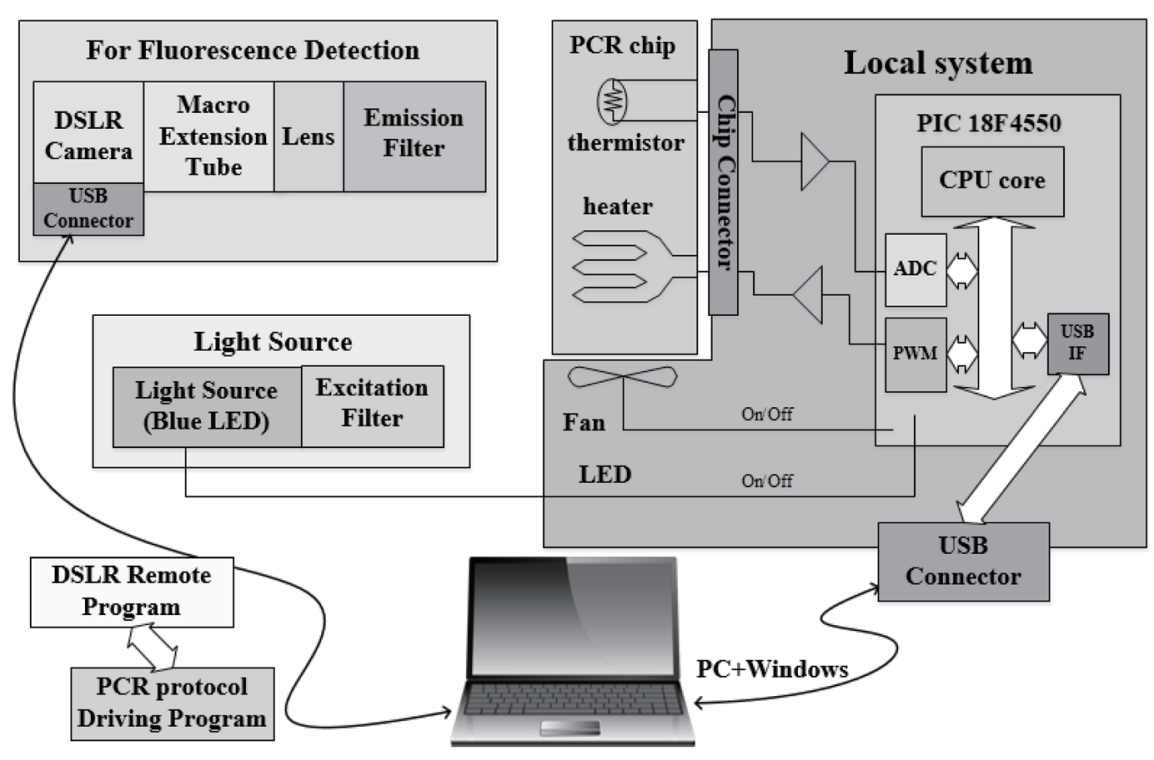

Fig. 1. Structure of camera-based fluorescence detection system. 


\subsection{RT-PCR Chips}

Figure 2 shows a PCR chip fabricated using a printed circuit board coated with four different materials used in the experiment. These coating materials are coated on the heating pattern of the PCB substrate, which is necessary for measuring the brightness of fluorescence during the RT-PCR test. The coating materials used are gold, silver, tin, and white silk, which were particularly selected because they can be coated on the printed circuit board.

In many previous experiments, when using a chip made of a general PCB substrate in RTPCR, light reflected on the chip surface acted as noise that lowers the overall signal-to-noise ratio when measuring the brightness of fluorescence. Therefore, in order to minimize light reflection, PCR was carried out using a chip made of a black matte substrate printed with a white silk legend on a heating pattern. However, since the color of the printed white silk legends was not constant, the deviation of the fluorescence brightness of the chips was very large.

In previous experiments, we used $50-\mu \mathrm{m}$-thick packing tape on top of the PCB substrate, $400-\mu \mathrm{m}$-thick black double-sided tape as a reaction chamber, and $500-\mu \mathrm{m}$-thick polycarbonate (PC) plastic film as a chip cover. ${ }^{(14)}$

Figure 3 shows the structure of the PCR chip used in the experiment, and a plastic housing was used to facilitate reagent injection and fix the chip. To compare fluorescence brightness deviations among the same type of chips, ten chips were manufactured for each type of coating using four kinds of PCB substrates having different coating materials.

The RT-PCR chips in Fig. 2 are the RT-PCR chips finally fabricated using a plastic housing. When distilled water and $1 \mathrm{ng} / \mu \mathrm{L}$ Chlamydia trachomatis (CT) DNA were amplified, 0.35 pmole/ $\mu \mathrm{L}$ FAM fluorescence reagent showing similar fluorescence brightness was injected into each chip. In the previous experiments, all the chips were injected with distilled water, then the distilled water was extracted, and the FAM reagent was injected and then photographed with a camera. In this experiment, it was difficult to analyze the same area when performing image analysis because the position of the chip was slightly changed since the chip was inserted into the connector to enable removal of the chip to extract the reagent and inject new reagent again. At the beginning of the experiment, the shooting conditions of the camera were ISO 1600 , aperture (AV) of 5.6, and shutter speed (TV) fixed at 1/10, 1/8, 1/6, 1/5, and 1/4 s.

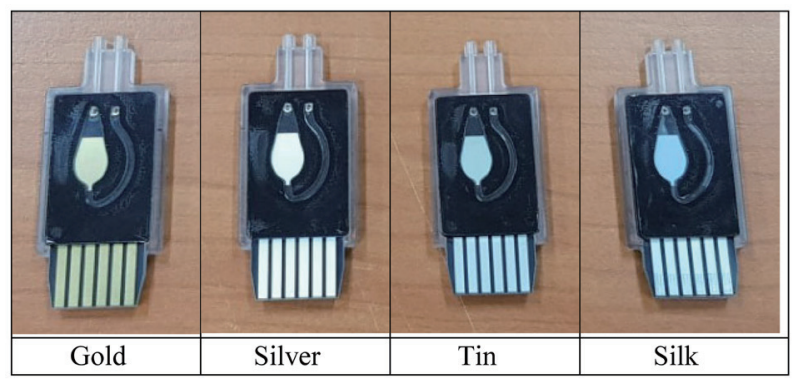

Fig. 2. (Color online) PCR chips used in this experiment. 


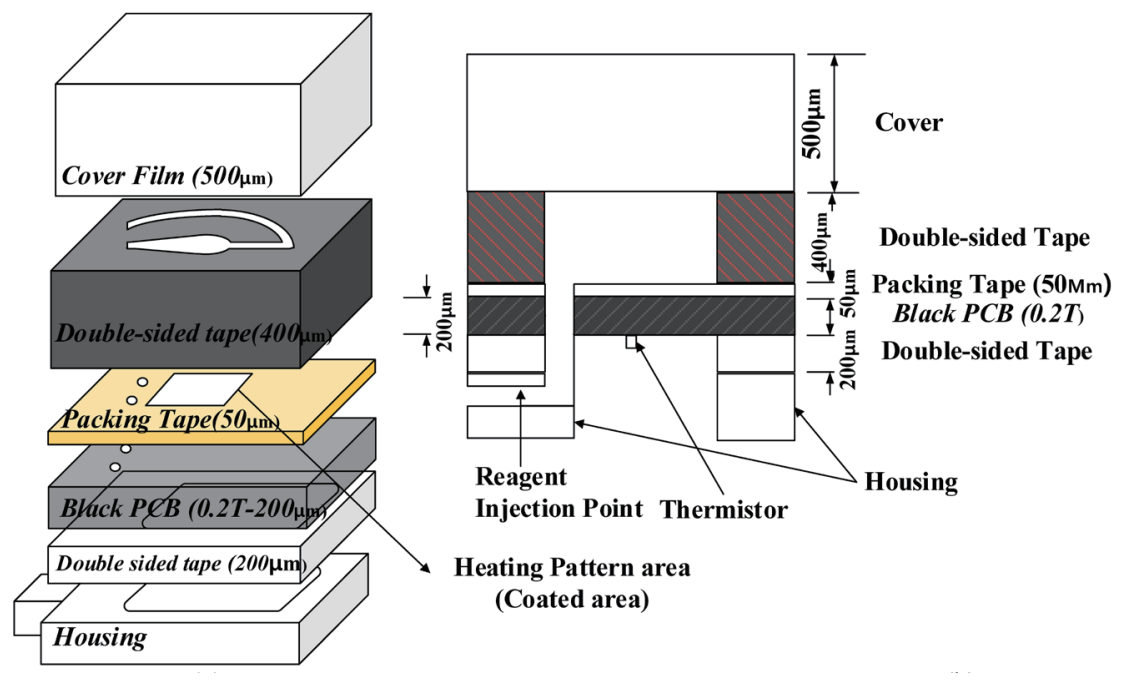

(a)

(b)

Fig. 3. (Color online) PCR chip structures: (a) assembly diagram and (b) cross section.

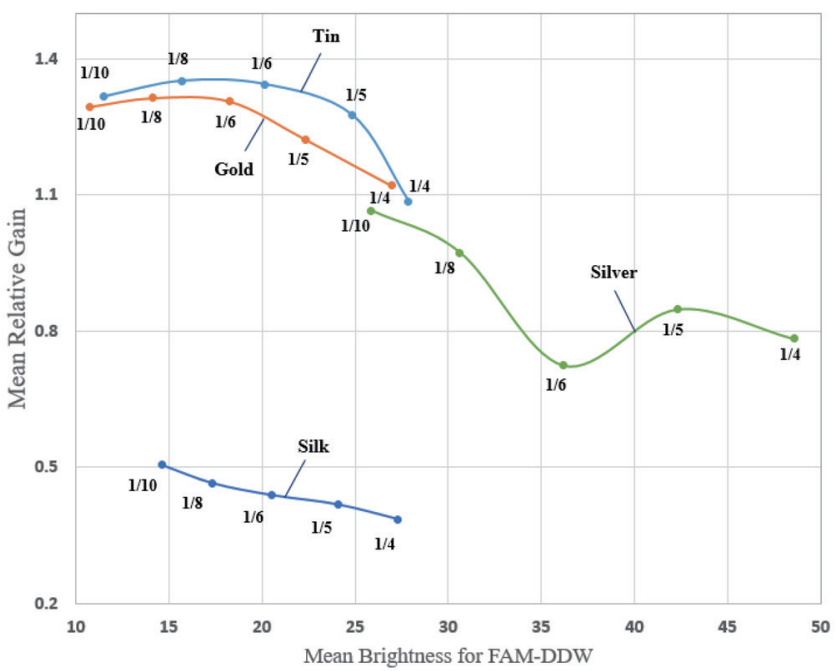

Fig. 4. (Color online) Average relative gain distribution based on FAM-DDW fluorescence average brightness in the first experiment.

However, as shown in Fig. 4, when the shutter speed is changed, the relative gain is gradually increased. When the brightness of the image is saturated, the value tends to decrease. This tendency was observed for gold and tin, but the test results of the chip coated with silk and silver showed different trends.

Therefore, when photographing a chip coated with silver and silk, the shutter speed was changed. The silk and silver coating chips were brightly photographed when shot at the same shutter speed as the tin and gold-coated chips, and some were shot at saturated brightness. So, we changed the shutter speed quickly and took pictures at 1/10,1/13,1/15, 1/20, and 1/25 s, which was darker than the previous experiment. 
As mentioned earlier, since the reagent was injected and photographed and the chip was detached from the connector, it was difficult to analyze the photographed images owing to the variation in the position of the chip, so the experiment procedure was changed in later experiments. After injecting the reagent and taking a picture with a camera, the chip was not removed from the connector, but the previously injected reagent was extracted and a new reagent was injected and then another picture was taken.

Figure 5 shows the fluorescence detection device used in the experiment. The chip was fixed as shown in the figure, and the blue LED was diagonally illuminated and captured with a DSLR camera (Canon 1100D).

In this work, we analyzed the images of 40 chips and compared the relative gain of fluorescence brightness for each coating material to select the best coating material on the chip.

\section{Experimental Results}

Table 1 shows the relative gain of the fluorescence images taken with the PCR chip made from the four types of PCBs with different coating materials and camera shutter speeds. In this work, the relative gain can be calculated using Eq. (1). To select the optimal chip material, subtract the average brightness of the chip that was injected with distilled water from the

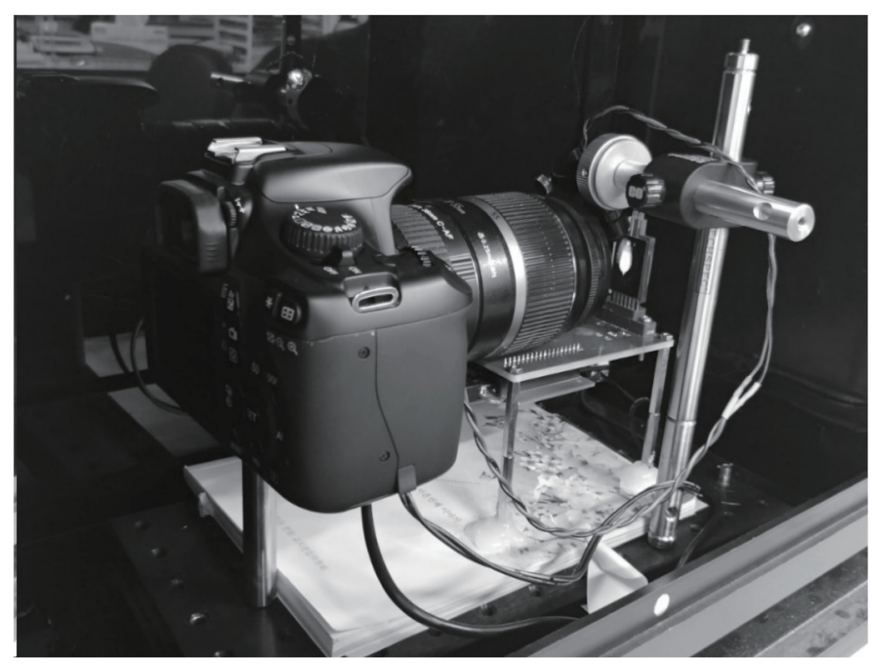

Fig. 5. Experimental environment of fluorescence detection.

Table 1

Relative gains of the four types of RT-PCR chips.

\begin{tabular}{lccccc}
\hline \multirow{2}{*}{ Material type } & \multicolumn{5}{c}{ Shutter speed } \\
\cline { 2 - 6 } & $1 / 10$ & $1 / 8$ & $1 / 6$ & $1 / 5$ & $1 / 4$ \\
\hline Gold & 0.956 & 1.034 & 1.035 & 1.014 & 0.999 \\
Tin & 1.105 & 1.115 & 1.099 & 1.004 & 0.877 \\
\hline \multirow{2}{*}{ Material type } & $1 / 25$ & $1 / 20$ & $1 / 15$ & $1 / 13$ & $1 / 10$ \\
\hline Silver & 1.076 & 1.115 & 1.451 & 1.091 & 1.073 \\
Silk & 0.551 & 0.558 & 0.549 & 0.499 & 0.441 \\
\hline
\end{tabular}


average chip brightness of the chip when the FAM fluorescent reagent was injected. Then, the value obtained by dividing this value by the average brightness of all chips injected with distilled water is called relative gain. The shutter speeds were the same for the gold and tin chips (1/10 to $1 / 4 \mathrm{~s})$ and the silver and silk chips (1/25 to $1 / 10 \mathrm{~s})$.

Regarding fluorescence brightness, the image was analyzed by changing the shutter speed and comparing the maximum relative gain. It was found that the gold-coated chip (hereinafter referred to as the gold chip) has the maximum relative gain when the shutter speed is $1 / 6 \mathrm{~s}$; for the silver-coated chip (hereinafter, the silver chip), $1 / 15 \mathrm{~s}$; for the silk legend printed chip (hereinafter, the silk chip), 1/20 s; and for the tin-coated chip (hereinafter, the tin chip), 1/8 s.

From the experimental results shown in Table 1, it can be seen that there is a difference in the relative gain depending on the type of material and the shutter speed of the camera.

$$
\text { Relative Gain }=[\text { Mean }(\text { FAM }- \text { DDW/DDW })]
$$

Table 2 shows the same type of chip-to-chip brightness deviation. In order to obtain the brightness deviation between chips, the coefficient of variation for the relative gain of each chip is calculated as shown by Eq. (2). If the PCB is uniformly coated, the brightness deviation between chips is small. As shown in Table 2, the gold chip has a small deviation.

$$
\mathrm{CV}=\{\mathrm{STDEV}(\mathrm{FAM}-\mathrm{DDW} / \mathrm{DDW}) / \mathrm{Mean}(\mathrm{FAM}-\mathrm{DDW} / \mathrm{DDW})\} \times 100
$$

Figure 4 shows the results of the previous experiment. It shows the change in the relative gain value with the shutter speed of the camera.

As shown in the figure, in the case of the gold and tin chips, the relative gain is largest when the shutter speed increases from $1 / 10$ to $1 / 8 \mathrm{~s}$ and then tends to decrease from $1 / 6 \mathrm{~s}$. Generally, the relative gain is calculated using the change in brightness. This relative gain value gradually increases and becomes maximum and thereafter, it tends to decrease. However, in the case of the silver and silk chips in Fig. 4, this tendency is not shown and only the decreasing tendency is shown. Therefore, it is unreasonable to select the optimum chip only on the basis of the experimental results. In other words, it was difficult to determine which coating material is optimal, so additional experiments were required.

Figure 6 shows the results of the analysis of the images of the brightness of the silk and silver chips photographed with the darker condition by increasing the shutter speed, unlike in previous

Table 2

Variation in brightness among four types of RT-PCR chips.

\begin{tabular}{lccccc}
\hline \multirow{2}{*}{ Material type } & \multicolumn{5}{c}{ Shutter speed } \\
\cline { 2 - 6 } & $1 / 10$ & $1 / 8$ & $1 / 6$ & $1 / 5$ & $1 / 4$ \\
\hline Gold & 4.562 & 3.362 & 6.083 & 6.680 & 6.457 \\
Tin & 6.397 & 5.499 & 6.322 & 7.029 & 8.176 \\
\hline \multirow{2}{*}{ Material type } & $1 / 25$ & $1 / 20$ & $1 / 15$ & $1 / 13$ & $1 / 10$ \\
\hline Silver & 8.000 & 9.718 & 10.611 & 10.142 & 11.133 \\
Silk & 25.257 & 26.203 & 31.306 & 34.360 & 30.766 \\
\hline
\end{tabular}




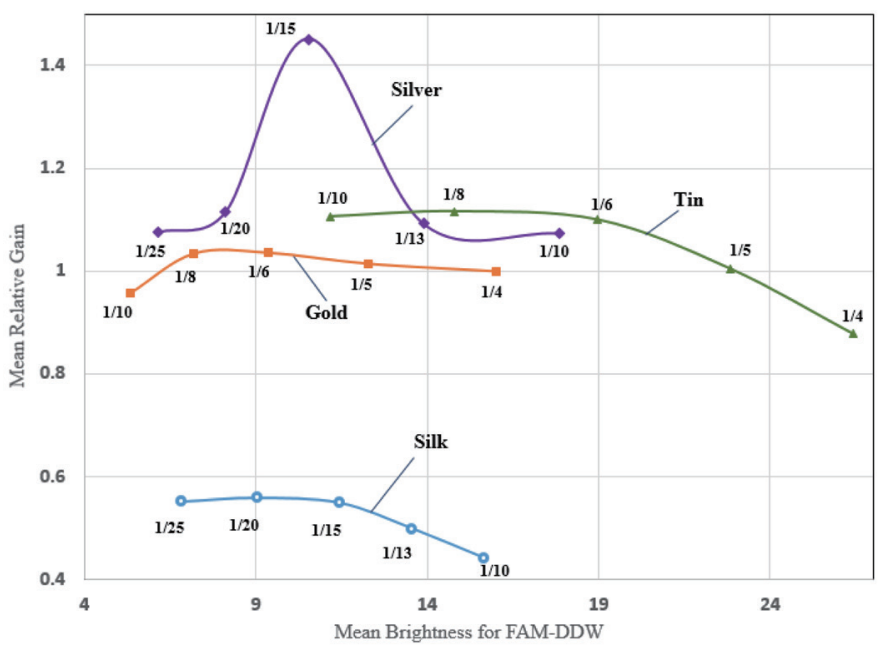

Fig. 6. (Color online) Average relative gain distribution based on FAM-DDW fluorescence average brightness in the final experiment.

experiments. For the gold and tin chips, the same shutter speeds as in the previous experiments were used, and the results of varying the experimental conditions showed that the maximum relative gain was obtained on the silk chip. The relative gain also increased, and after peaking, it showed a tendency to decrease again.

In this figure, the analysis was performed with different shutter speeds, but the relative gain was found to be the largest for silver chips, decreasing in the order of tin chips and gold chips, and lastly, the silk chips. According to the experimental results, if the chip is fabricated with a silver-coated substrate and RT-PCR is performed, much more reliable experimental results can be expected to be obtained. However, biocompatibility is also important for PCR. In this experiment, the FAM reagent with the same fluorescent intensity as that at the end of DNA amplification was used without actual samples. Therefore, it is necessary to examine the possibility of actual DNA amplification, as well as the reliability at the time of fluorescence detection.

\section{Conclusions}

In previous studies, the RT-PCR chip used for the quantitative analysis of RT-PCR was a white silk legend printed on a heating pattern on top of a black matte PCB. On top of that was attached packing tape, a black double-sided tape, and a PC film. When the result of fluorescence detection was analyzed using the RT-PCR chip thus produced, the color of the printed silk was uneven and the relative gain was small, and there was the problem that the deviation of the fluorescence brightness between the chips was large.

Therefore, in this study, we made 10 PCR chips for each type using four kinds of materials that can be coated on the PCB substrate. Each chip was injected with distilled water and a 0.35 pmole/ $\mu \mathrm{L}$ FAM reagent; then, a fluorescence brightness similar to that when DNA amplification was complete was exhibited. The shutter speed setting of the camera was varied and the chips were photographed. Then we compared the fluorescence brightness in the images. 
The images captured at different shutter speeds were analyzed for the four types of chips, and the relative gains in each image were calculated and compared. In an experiment with gold and tin chips, the camera shutter speed was set at 1/10,1/8,1/6, 1/5, and 1/4 s, and images were taken. In the case of the silver and silk chips, the shutter speed was set at 1/25, 1/20, 1/15, 1/13, and $1 / 10 \mathrm{~s}$. In the first experiment, since the maximum relative gain of the silver and silk chips could not be obtained, the maximum relative gain was obtained by increasing the shutter speed. Also, the tendency of the change in relative gain with the change in the shutter speed was also similar between the silver and silk chips.

As a result of analyzing the relative gain values of the four types of chips, that of the silver chip was found to be the highest, followed by those of the tin and gold chips; the silk chip showed the lowest relative gain. It was determined that the chip with a good signal-to-noise ratio is the silver chip. However, since the fluorescence reagent of the same brightness was used instead of an actual sample in this experiment, an additional biocompatibility test of PCR using an actual sample is necessary.

\section{Acknowledgments}

This research was supported by the National Research Foundation of Korea (NRF) funded by the Ministry of Science, ICT and Future Planning (NRF-2017T1A2B1010125).

\section{References}

1 D. Patko, Z. Mártonfalvi, B. Kovacs, F. Vonderviszt, M. Kellermayer, and R. Horvath: Sens. Actuators, B 196 (2014) 352.

2 C. A. Heid, J. Stevens, K. J. Livak, and P. M. Williams: Genome Res. 6 (1996) 986.

3 D. Klein: Trends Mol. Med. 8 (2002) 257.

4 S. Bustin, V. Benes, T. Nolan, and M. Pfaffl: J. Mol. Endocrinol. 34 (2005) 597.

5 J. S. Hwang, J. D. Kim, Y. S. Kim, H. J. Song, and C. Y. Park: Int. J. Control Autom. 8 (2015) 117.

6 J. S. Hwang, J. D. Kim, Y. S. Kim, H. J. Song, and C. Y. Park: Asia Life Sci. 12 (2015) 499.

7 J. S. Hwang, Y. S. Kim, H. J. Song, J. D. Kim, and C. Y. Park: Technol. Health Care 24 (2016) S139.

8 C. Y. Park, J. D. Kim, J. H. Ku, Y. S. Kim, H. J. Song, and J. Kim: Sens. Lett. 10 (2012) 1197.

9 K. Shen, X. Chen, M. Guo, and J. Cheng: Sens. Actuators, B 105 (2005) 251.

10 T. M. Hsieh, C. H. Luo, F. C. Huang, J. H. Wang, L. J. Chien, and G. B. Lee: Sens. Actuators, B 130 (2008) 848.

11 J. H. Wang, L. J. Chien, T. M. Hsieh, C. H. Luo, W. P. Chou, P. H. Chen, P. J. Chen, D. S. Lee, and G. B. Lee: Sens. Actuators, B 141 (2009) 329.

12 C. E. Wills: Electro/94 Int. Conf. Combined Volumes (1994) 415.

13 J. Kim, J. Kim, and G. Lee: SPIE MOEMS-MEMS 7929 (2011) 79290Y.

14 J. S. Hwang, J. D. Kim, Y. S. Kim, H. J. Song, and C. Y. Park: Sens. Mater. 30 (2018) 397. 